\title{
Effet de la salinité et de la restriction de l'eau d'abreuvement sur les métabolites sanguins des caprins Arbia en Algérie
}

\author{
Zeyneb Mehalaine ${ }^{1}$ Kahina Chaker-Houd ${ }^{2}$ Abdelaziz Nadir \\ Ghamri $^{3}$ Lamia Mebirouk-Boudechiche ${ }^{1 *}$ Saida Matallah ${ }^{1}$
}

\begin{abstract}
Mots-clés
Caprin, eau saline, privation d'eau, métabolite, Algérie
\end{abstract}

Submitted: 8 January 2018

Accepted: 1 October 2018

Published: 14 January 2019

DOI: $10.19182 /$ remvt.31667

\begin{abstract}
Résumé
L'étude visait à déterminer l'effet du stress hydrique sur les métabolites sanguins des caprins de race Arbia en Algérie. Quatre groupes de dix boucs chacun ont été aléatoirement soumis à des régimes d'abreuvement différents : le groupe 1 (témoin) avait libre accès à une eau de source (trois litres), tandis que les groupes 2 et 3 recevaient la même eau enrichie respectivement de deux et cinq grammes de chlorure de sodium par litre. Le groupe 4 avait uniquement accès à $25 \%$ de la quantité d'eau distribuée aux boucs du groupe témoin. A la fin de la période expérimentale, des prélèvements sanguins ont été réalisés afin de doser les protéines totales, la créatinine, l'albumine, l'urée, le cholestérol total et le glucose. Les différents traitements ont augmenté les concentrations plasmiques de l'albumine, de la créatinine, des protéines totales et du cholestérol total, ainsi que l'urée des animaux ayant subi une restriction hydrique. Cependant, ils n'ont pas affecté la glycémie. Ces résultats ont montré que l'exposition à court terme (12 jours) de boucs Arbia à la salinisation de l'eau d'abreuvement ou à une restriction hydrique affectait la majorité de leurs métabolites sanguins à l'exception du glucose.
\end{abstract}

- Pour citer cet article : Mehalaine Z., Chaker-Houd K., Ghamri A.N., Mebirouk-Boudechiche L., Matallah S., 2018. Effect of salinity and water restriction on blood metabolites of Arbia goats in Algeria. Rev. Elev. Med. Vet. Pays Trop., 71 (4): 167-172, doi: 10.19182/remvt.31667

\section{INTRODUCTION}

La population caprine en Algérie a été estimée à environ 4,9 millions de têtes en 2011 (FAO, 2014, cité par Sahi et al., 2018), répartie principalement dans les zones montagneuses dans lesquelles elle est bien adaptée. L'élevage caprin est l'une des plus importantes activités agricoles en milieu rural car ces animaux sont source de protéines à haute valeur biologique comme le lait et la viande. Ils sont considérés

\footnotetext{
1. Laboratoire d'épidémio-surveillance, santé, productions et reproduction, expérimentation et thérapie cellulaire des animaux domestiques et sauvages, Université Chadli Bendjedid El Tarf, BP 73, 36000 El Tarf, Algérie.

2. Laboratoire d'agriculture et fonctionnement des écosystèmes, Université Chadli Bendjedid El Tarf, 36000 El Tarf, Algérie.

3. Laboratoire santé animale, productions végétales, environnement et sécurité alimentaire, Université Chadli Bendjedid El Tarf, 36000 El Tarf, Algérie.

* Auteur pour la correspondance

E-mail : boudechiche.lamia@gmail.com
}

comme étant adaptés au manque d'eau et d'aliments. Ils ont la capacité de tirer profit des ressources du milieu pastoral dans lequel ils se trouvent et de tolérer des environnements difficiles, notamment les régions arides et semi-arides, considérées comme défavorables aux autres ruminants domestiques (Silanikove, 2000). Les faibles précipitations dans ces régions affectent la disponibilité en eau et en aliments. L'eau est souvent un facteur limitant pour les caprins qui vivent dans ces zones, où sa salinisation constitue, en outre, une contrainte compromettant l'efficacité et la durabilité des systèmes d'élevage (Wilson, 1975). Cette situation risque d'être aggravée dans les années à venir par le changement climatique auquel fait face le monde.

L'eau joue un rôle majeur chez les ruminants, car elle influence de manière critique les modèles digestifs et métaboliques ainsi que les performances productives. Par conséquent, des informations sur les besoins en eau et les réponses du bétail aussi bien à la qualité (salinité) qu'à la quantité consommée sont nécessaires pour évaluer l'efficacité des pratiques de consommation au niveau de l'exploitation et développer des stratégies d'abreuvement appropriées qui améliorent la performance du bétail et le garde en bonne santé. Ceci est particulièrement pertinent dans les régions arides et semi-arides. 
La salinité de l'eau exprime la quantité de solides dissous dans l'eau. Souvent ce sont des sels minéraux communs du sol comme les chlorures de sodium $(\mathrm{NaCl})$, de magnésium $(\mathrm{Mg})$ et de calcium $(\mathrm{Ca})$, les carbonates, bicarbonates, sulfates et phosphates ; mais d'autres substances comme les nitrates, les nitrites et les oligoéléments peuvent également être présents à des concentrations variant avec la source d'eau (Yousfi et Ben Salem, 2017). Les ruminants présentent différentes réponses comportementales et physiologiques à l'eau salée.

Casamassima et al. (2016) et Grech-Angelini (2007) montrent comment des ruminants (ovins, bovins, caprins ou camelins) répondent au stress hydrique (déshydratation ou niveau d'hydratation), et Yousfi et Ben Salem (2017) et Eltayeb (2000) montrent leurs réponses selon le niveau de salinité de l'eau d'abreuvement. Les réponses dépendent des espèces animales, de leur état physiologique, des conditions climatiques, du régime alimentaire et du niveau de salinité de l'eau.

Ce type d'étude n'a pas été mené sur les caprins de race locale en Algérie. Ainsi ce travail propose d'étudier les effets du stress hydrique, aussi bien l'augmentation des niveaux de sels que la restriction hydrique, sur le profil sanguin des caprins de race locale connus pour leur adaptation aux conditions difficiles prévalant dans les régions semi-arides et arides.

\section{MATERIEL ET METHODES}

\section{Zone d'étude}

L'étude a été réalisée dans la commune d'Oum Laadhaim située au sud-ouest de la wilaya de Souk Ahras, elle-même située au nord-est de l'Algérie, à 640 kilomètres d'Alger ( $36^{\circ} 10^{\prime} \mathrm{N}$ et $\left.7^{\circ} 55^{\prime} \mathrm{E}\right)$. Elle est limitrophe au nord des wilayas d'El Tarf et de Guelma, à l'ouest de celle d'Oum El Bouaghi, au sud de celle de Tébessa, à l'est de la Tunisie. Située sur les hauteurs de l'Atlas tellien, Souk Ahras est exposée aux influences climatologiques méditerranéennes avec un climat méditerranéen, subhumide au nord et semi-aride à l'extrême sud. La moyenne annuelle des précipitations est de 650 millimètres au nord et de 350 au sud. Les températures moyennes sont de $7,9^{\circ} \mathrm{C}$ en janvier et $27,6^{\circ} \mathrm{C}$ en juillet.

\section{Animaux, régimes alimentaires et dispositif expérimental}

L'expérience a porté sur 40 boucs de race locale Arbia et de même âge (2-3 ans, $50 \mathrm{~kg}$ en moyenne), répartis durant la saison d'été (août) en quatre lots homogènes :

- lot 1 (témoin), les animaux ont eu accès ad libitum à l'eau d'abreuvement issue d'une source. Les mesures quotidiennes durant la phase préexpérimentale ont permis d'estimer l'eau volontairement consommée à trois litres par animal par jour ;

- lot 2, les animaux ont eu accès à la même eau d'abreuvement que le lot 1 mais enrichie de $2 \mathrm{~g}$ de $\mathrm{NaCl}$ par litre (faible salinité) ;

- lot 3, les animaux ont eu accès à la même eau d'abreuvement que le lot 1 mais enrichie de $5 \mathrm{~g}$ de $\mathrm{NaCl} / \mathrm{L}$ (forte salinité);

- lot 4 , les animaux ont eu accès uniquement à $25 \%$ de la quantité d'eau consommée par les animaux du lot témoin (restriction hydrique).

Une période d'adaptation de sept jours a été suivie par une période expérimentale de 12 jours au cours de laquelle les animaux, conduits en système extensif, ont profité des parcours arbustifs de chêne Kermès (Quercus coccifera), d'armoise commune (Artemisia vulgaris), de romarin (Rosmarinus officinalis) et de deremeth (Haloxylon scoparium). Ils pâturaient sur les chaumes de céréales et à leur retour du pâturage ils recevaient de l'orge concassée à raison de 400 grammes par animal par jour et du foin de prairie à volonté. Ils pâturaient quotidiennement pendant six heures (de $7 \mathrm{~h} 30$ à $11 \mathrm{~h}$ le matin et de $15 \mathrm{~h} 30$ à $18 \mathrm{~h}$ l'aprèsmidi). Au retour du pâturage, les animaux de chaque lot étaient placés dans des box séparés, dans lesquels des abreuvoirs contenaient l'eau d'abreuvement qui leur était spécifique. Ils avaient facilement accès à l'eau qu'ils recevaient ad libitum, sauf le lot 4 .

\section{Analyses physico-chimiques de l'eau d'abreuvement}

Dans le but d'estimer la qualité de l'eau d'abreuvement de chaque lot d'animaux, des analyses physico-chimiques ont été réalisées portant sur la détermination du $\mathrm{pH}$ à l'aide d'un $\mathrm{pH}$ mètre électronique, de la conductivité, de la salinité et des solides dissous totaux (TDS) à l'aide d'un conductivimètre à électrode. Le calcium et la turbidité ont été analysés selon la méthode titrimétrique à l'acide éthylène diamine tétra acétique (EDTA) (respectivement, ISO 1984 ; 1990), la dureté totale selon les normes ISO (1984).

\section{Analyses biochimiques}

A la fin de la période expérimentale, des prélèvements sanguins ont été réalisés par ponction de la veine jugulaire à $7 \mathrm{~h}$ du matin avant la prise alimentaire. Les échantillons sanguins ont été collectés dans des tubes stériles héparinés et envoyés au laboratoire d'analyses. Les plasmas correspondants ont été conservés à $-20{ }^{\circ} \mathrm{C}$ afin d'évaluer certains paramètres biochimiques sanguins des animaux soumis au stress hydrique. L'urée a été déterminée par la méthode de Berthelot (1860) utilisant l'EDTA, l'albumine par celle de Young et Friedman (2001) en utilisant le vert de bromocrésol colorimétrique, et la créatinine par celle de Murray (1984). La technique pour le dosage du cholestérol a été celle de Richmond (1973) en utilisant de l'oxydase cholestérol suivant la saponification des échantillons, tandis que les protéines totales ont été déterminées par la méthode du réactif de Biuret de King et Wooton (1965). Enfin le glucose a été dosé par la méthode de Trinder (1969).

\section{Analyses statistiques}

Les données obtenues concernant les métabolites sanguins en fonction du régime hydrique ont été soumises à une analyse de la variance (Anova) à un seul facteur selon la procédure du modèle linéaire généralisé (GLM) du logiciel SAS (2004). La différence entre les moyennes a été testée par la procédure LSMEANS. Le test de Turkey a été réalisé pour la comparaison multiple des moyennes au seuil de signification de $5 \%$.

\section{RESULTATS}

\section{Qualité physico-chimique des eaux d'abreuvement}

Les analyses physico-chimiques de l'eau ont révélé des variations au niveau de l'ensemble des paramètres étudiés sauf la température qui n'a présenté aucune variation, soit une température de $20{ }^{\circ} \mathrm{C}$ pour l'ensemble des échantillons. En revanche, pour la turbidité, les valeurs ont été comprises entre 1,02 et 1,55 NTU. Néanmoins, les valeurs les plus importantes de ce paramètre ont été enregistrées au niveau des échantillons d'eaux traitées au $\mathrm{NaCl}$ (tableau I).

Les résultats ont révélé un pH oscillant entre 7,42 et 8,14. L'examen de la conductivité a révélé une importante variation allant de 451 à 774 $\mu \mathrm{S} / \mathrm{cm}$. Toutefois, la conductivité de l'eau non traitée était largement inférieure à celles des eaux traitées. La dureté mesurée de l'eau sans traitement était de $17,8^{\circ} \mathrm{F}$, alors que des diminutions de 3,5 et $4,3{ }^{\circ} \mathrm{F}$ ont été enregistrées respectivement avec l'apport de 2 et $5 \mathrm{~g} / \mathrm{L}$ de $\mathrm{NaCl}$. Parallèlement à la dureté, une variation a aussi été enregistrée pour le calcium avec un maximum de $116,87 \mathrm{mg} / \mathrm{L}$ et un minimum de $96,72 \mathrm{mg} / \mathrm{L}$. Le taux de sels dissous a augmenté significativement de $307 \mathrm{mg} / \mathrm{L}$ jusqu'à $3780 \mathrm{mg} / \mathrm{L}$ avec le traitement avec ajout de $\mathrm{NaCl}$. 


\section{Analyses biochimiques}

Le tableau II présente les concentrations sanguines de glucose et de cholestérol chez les caprins Arbia en fonction de la nature de l'eau d'abreuvement. Si la glycémie n'a pas été significativement affectée par la forte salinité de l'eau, la cholestérolémie a augmenté, aussi bien chez les caprins ayant reçu une eau enrichie de $5 \mathrm{~g} / \mathrm{L}$ que chez ceux ayant subi une restriction hydrique de $25 \%(\mathrm{p}=0,00)$. Cette dernière a semblé affecter la glycémie dont les valeurs ont été supérieures aux normes $(0,95 \mathrm{~g} / \mathrm{L})$.

Le tableau II montre aussi l'effet de la salinité de l'eau d'abreuvement et de la restriction hydrique chez les caprins Arbia sur les protéines totales plasmatiques, l'albumine et la créatinine. Les concentrations en créatinine et en protéines totales ont augmenté significativement avec la salinité de l'eau : avec une eau à $5 \mathrm{~g} / \mathrm{L}$, respectivement 15,43 vs $20,37 \mathrm{mg} / \mathrm{L}$ et 72,9 vs $81,36 \mathrm{~g} / \mathrm{L}$; avec la restriction hydrique, respectivement 15,43 vs $24,33 \mathrm{mg} / \mathrm{L}$ et 72,9 vs $89,20 \mathrm{~g} / \mathrm{L}$.

Il en était de même pour l'albumine qui était élevée dans les lots avec ajout de sel à 2 et $5 \mathrm{~g} / \mathrm{L}$, ou ayant subi une restriction hydrique (respectivement 40,65, 41,38 g/L et $41 \mathrm{~g} / \mathrm{L})$ par rapport au témoin $(38,16 \mathrm{~g} / \mathrm{L})$. La salinité de l'eau n'a pas influencé de façon significative l'urémie qui a, en revanche, été significativement affectée par la restriction hydrique enregistrant des valeurs élevées $(0,77 \mathrm{~g} / \mathrm{L})$.

\section{DISCUSSION}

\section{Qualité physico-chimique de l'eau d'abreuvement}

Les propriétés physico-chimiques de l'eau peuvent aider à déterminer la qualité de l'eau. Elles incluent le $\mathrm{pH}$, les solides dissous totaux et la dureté (Beede, 2005). Cependant, « au cours de leur circulation souterraine et de leur séjour dans l'aquifère, les eaux des nappes phréatiques se chargent, au contact des différents terrains traversés, d'un certain nombre d'éléments chimiques solubles. Ces éléments sont d'origine géologique le plus souvent ; ils peuvent cependant parfois être d'origine anthropique et sont alors susceptibles d'affecter sensiblement la qualité de l'eau » (Rodier et al., 2009).

La température de l'eau est un facteur important dans la production biologique (HCEFLCD, 2006). Cependant, elle peut avoir une réelle influence sur sa consommation (Olkowski, 2009). Lorsque la température de l'eau baisse, sa consommation diminue et les animaux tolèrent alors mieux une concentration relativement plus élevée en contaminants (El Moustaine et al., 2013). Par ailleurs, les températures des sources analysées sont relativement moyennes et restent dans la normalité des eaux d'abreuvement des caprins. D'ailleurs, chez les caprins l'ingestion d'eau augmente lorsque la température extérieure subit une hausse afin de combler leurs besoins.

Tableau I

Paramètres physico-chimiques de l'eau d'abreuvement des caprins de race locale Arbia en Algérie

\begin{tabular}{lcccccccc} 
Nature de l'eau & $\begin{array}{c}\mathbf{T} \\
\left({ }^{\circ} \mathbf{C}\right)\end{array}$ & $\begin{array}{c}\text { Turbidité } \\
(\mathbf{N T U})\end{array}$ & $\mathbf{p H}$ & $\begin{array}{c}\text { Conductivité } \\
(\boldsymbol{\mu} \mathbf{S} / \mathbf{c m})\end{array}$ & $\begin{array}{c}\text { TH } \\
(\mathbf{F})\end{array}$ & $\begin{array}{c}\mathbf{C a}^{2+} \\
(\mathbf{m g} / \mathbf{L})\end{array}$ & $\begin{array}{c}\text { TDS } \\
(\mathbf{m g} / \mathbf{L})\end{array}$ & $\begin{array}{c}\text { Salinité } \\
(\mathbf{N a C l} \mathbf{g} / \mathbf{L})\end{array}$ \\
\hline Eau de source & 20 & 1,02 & 7,42 & 451 & 17,8 & 116,87 & 307 & 0,4 \\
Eau de source $(+2 \mathrm{~g} \mathrm{NaCl})$ & 20 & 1,55 & 8,14 & 646 & 14,3 & 112,84 & 2440 & 2,2 \\
Eau de source $(+5 \mathrm{~g} \mathrm{NaCl})$ & 20 & 1,46 & 7,99 & 774 & 13,5 & 96,72 & 3780 & 5,7 \\
Normes (OMS) & - & $<5$ & $6,5-9,2$ & $<2100$ & $<50$ & $<250$ & 1000 & -
\end{tabular}

$\mathrm{T}$ : température ; NTU : unité néphélométrique de turbidité ; $\mathrm{pH}$ : potentiel hydrogène ; $\mu S:$ microsiemens ; TH : titre hydrotimétrique, dureté totale ; F : degré français ; TDS : taux de sels dissous totaux ; OMS : Organisation mondiale de la santé

\section{Tableau II}

Effet de la salinité de l'eau et de la restriction hydrique sur des paramètres biochimiques sanguins des caprins de race locale Arbia en Algérie

\begin{tabular}{|c|c|c|c|c|c|c|}
\hline & $\begin{array}{l}\text { Glucose } \\
(\mathrm{g} / \mathrm{L})\end{array}$ & $\begin{array}{c}\text { Cholestérol total } \\
(\mathrm{g} / \mathrm{L})\end{array}$ & $\begin{array}{c}\text { Protéines totales } \\
(\mathrm{g} / \mathrm{L})\end{array}$ & $\begin{array}{l}\text { Albumine } \\
(\mathrm{g} / \mathrm{L})\end{array}$ & $\begin{array}{c}\text { Urée } \\
(\mathbf{m m o l} / \mathrm{L})\end{array}$ & $\begin{array}{l}\text { Créatinine } \\
(\mathrm{mg} / \mathrm{L})\end{array}$ \\
\hline Lot 1 & $0,80^{\mathrm{a}} \pm 0,22$ & $0,65^{\mathrm{C}} \pm 0,09$ & $72,90^{d} \pm 3,01$ & $38,16^{b} \pm 2,92$ & $12^{b} \pm 0,17$ & $15,43^{\mathrm{C}} \pm 2,79$ \\
\hline Lot 2 & $0,80^{\mathrm{a}} \pm 0,10$ & $1,02^{b} \pm 0,11$ & $77,36^{c} \pm 2,98$ & $40,65^{a} \pm 2,39$ & $10^{c} \pm 0,09$ & $17,12^{\mathrm{c}} \pm 2,20$ \\
\hline Lot 3 & $0,79^{a} \pm 0,14$ & $1,44^{\mathrm{a}} \pm 0,33$ & $81,36^{b} \pm 2,22$ & $41,38^{a} \pm 2,04$ & $9,8^{\mathrm{c}} \pm 0,07$ & $20,37^{b} \pm 1,65$ \\
\hline Lot 4 & $0,90^{\mathrm{a}} \pm 0,20$ & $1,52^{\mathrm{a}} \pm 0,22$ & $89,20^{a} \pm 2,53$ & $41^{\mathrm{a}} \pm 2,93$ & $12,8^{\mathrm{a}} \pm 0,09$ & $24,33^{\mathrm{a}} \pm 3,01$ \\
\hline$P$ & $\begin{array}{l}0,0874 \\
\text { NS }\end{array}$ & $\begin{array}{l}0,0001 \\
* * *\end{array}$ & $\begin{array}{l}0,0001 \\
* * *\end{array}$ & $\begin{array}{l}0,2711 \\
\text { NS }\end{array}$ & $\begin{array}{l}0,0008 \\
* * *\end{array}$ & $\begin{array}{l}0,0001 \\
* * *\end{array}$ \\
\hline \multirow[t]{2}{*}{ Valeurs usuelles } & $0,45-0,82$ & $0,80-1,30$ & $62-79$ & $26-38$ & $7-12$ & $6-16$ \\
\hline & $\begin{array}{l}\text { Grech-Angelini } \\
(2007)\end{array}$ & $\begin{array}{l}\text { Zabre } \\
\text { (2013) }\end{array}$ & $\begin{array}{c}\text { Jackson et } \\
\text { Cockcroft (2002) }\end{array}$ & $\begin{array}{c}\text { Jackson et } \\
\text { Cockcroft (2002) }\end{array}$ & $\begin{array}{l}\text { Kaneko et al., } \\
\text { 2008, cités par } \\
\text { Kouamo et al., } 2014\end{array}$ & $\begin{array}{c}\text { Jackson et } \\
\text { Cockcroft (2002) }\end{array}$ \\
\hline
\end{tabular}

Lot 1 : témoin ; lot 2 : ajout de $2 \mathrm{~g} \mathrm{NaCl} / \mathrm{L}$; lot 3 : ajout de $5 \mathrm{~g} \mathrm{NaCl} / \mathrm{L}$; lot $4: 25 \%$ de restriction hydrique par rapport au témoin

$\mathrm{a}, \mathrm{b}, \mathrm{c}, \mathrm{d}$ Différentes lettres sur une même colonne indiquent une différence significative entre les métabolites sanguins $(\mathrm{p}<0,05) ; * * *$ Différence très significative au seuil de $5 \%$; NS : non significatif 
La turbidité donne des informations visuelles sur l'eau relatives au degré de transparence et de propreté qui dépend de la qualité des matières en suspension (argiles, débris organiques, organismes microscopiques (Rodier et al., 2009). La turbidité de l'ensemble des eaux d'abreuvement analysées était peu importante ; les eaux étaient incolores et répondaient aux normes de l'Organisation mondiale de la santé (5 NTU ; OMS) pour un tel usage ; ceci était probablement dû à l'efficacité du processus de filtration par les différents horizons du substrat pédologique traversé (Gouaidia, 2008). Toutefois, l'un des facteurs pouvant augmenter les valeurs de la turbidité sont les particules en suspension sur lesquelles se fixent généralement les microorganismes dont certains sont capables d'affecter la santé animale (Olkowski, 2009).

Le $\mathrm{pH}$ des eaux dépend de leurs origines, de la nature géologique du substrat et du bassin versant traversé (Gouaidia, 2008 ; Rodier et al., 2009). Ce paramètre peut avoir des répercussions plus importantes sur les animaux d'élevage en augmentant les risques d'acidose ou d'alcalose métabolique (Olkowski, 2009). Néanmoins, les valeurs observées ont révélé que le pH des eaux analysées était neutre avec une légère tendance alcaline pour celles traitées avec le $\mathrm{NaCl}$; pourtant, ces eaux restaient dans les limites de potabilité pour les caprins.

La conductivité constitue un bon indice de minéralisation de l'eau. Les valeurs mesurées ont révélé une minéralisation moyenne pour les eaux non traitées et une forte minéralisation pour les échantillons traités avec le $\mathrm{NaCl}$. Néanmoins, les résultats de l'ensemble des eaux analysées peuvent être considérés comme acceptables.

D’après Rodier et al. (2009), une valeur élevée des TDS signifie une grande quantité de sels en solution. Ceci concorde avec nos résultats montrant que l'augmentation de la concentration en $\mathrm{NaCl}$ avait entraîné une augmentation des TDS. Néanmoins, les eaux traitées présentaient des concentrations en sels supérieures aux normes de l'OMS pour l'abreuvement des caprins.

La dureté totale d'une eau est produite essentiellement par les sels de calcium et de magnésium qu'elle contient (Belghiti et al., 2013). Les résultats ont révélé que les eaux d'abreuvement analysées étaient douces (titre hydrotimétrique $[\mathrm{TH}]<15^{\circ} \mathrm{F}$ ), sauf pour l'eau de source non traitée qui a eu tendance à être plutôt dure tout en étant dans les normes $\left(15<\mathrm{TH}<30^{\circ} \mathrm{F}\right)$. Ces résultats sont probablement liés à la nature lithologique de la formation. Cependant, une dureté totale faible $\left(\mathrm{TH}<15^{\circ} \mathrm{F}\right)$ peut engendrer chez les petits ruminants (ovins) des diarrhées et une coloration de la viande, alors qu'une forte dureté $\left(\mathrm{TH}>15^{\circ} \mathrm{F}\right.$ ) provoque une anémie (AAVL, 2006).

\section{Paramètres plasmatiques du métabolisme énergétique}

Dans notre étude, la salinité de l'eau d'abreuvement n'a pas eu d'effet significatif sur le niveau de glucose plasmatique chez les caprins de race locale, comme rapporté par Eltayeb (2000) chez des chèvres Nubiennes. D'autres auteurs ont fait le même constat chez les ovins (Casamassima, 2016) comme chez les dromadaires (Grech-Angelini, 2007). En revanche, Yousfi et Bensalem (2017) rapportent une augmentation du glucose sanguin chez des moutons Barbarins abreuvés avec une eau fortement saline.

Dans la présente étude, une augmentation progressive de la cholestérolémie a été enregistrée à mesure que l'eau d'abreuvement devenait saline. Cette augmentation a été plus importante chez les animaux soumis à une restriction hydrique de $25 \%$, comme rapporté par Casamassima et al. (2008) chez des ovins Comisana soumis à une restriction hydrique. En revanche, Casamassima et al. (2016) ne rapportent aucun effet de la restriction hydrique sur la cholestérolémie chez les brebis Lacaunes. Ils attribuent ces résultats contradictoires à l'effet de la race et au fait que les ovins diffèrent dans leurs capacités respectives à répondre à la restriction de l'eau. Chez le dromadaire, la déshydratation aurait tendance à augmenter le taux de cholestérol sérique (Grech-Angelini, 2007).

\section{Paramètres plasmatiques du métabolisme azoté}

La protéinémie moyenne est apparue plus élevée dans la présente étude que celle rapportée par Jackson et Cockcroft (2002), aussi bien chez les caprins ayant subi une restriction hydrique que chez ceux ayant reçu une eau salée à $5 \mathrm{~g} / \mathrm{L}$. Le même constat est fait par Hadjigeorgiou et al. (2000) pour l'eau salée. Gomaa (1996) rapporte que les concentrations des protéines sériques et leurs fractions augmentent avec la déshydratation chez tous les animaux. En effet, en raison de leur poids moléculaire élevé, les transferts de protéines vers les autres milieux liquidiens sont très faibles, par conséquent, toute diminution du volume plasmatique entraine une augmentation de leur concentration.

Houpt (2004), cité par Eltayeb (2000), indique que lorsqu'une solution hypertonique de $\mathrm{NaCl}$ est administrée par voie intraveineuse chez le mouton, l'eau se déplace dans le plasma et l'osmolarité accrue du liquide extracellulaire provoquerait la déshydratation cellulaire. L'augmentation du taux de protéines totales parallèlement à la salinité de l'eau dénote une corrélation positive entre les protéines totales du plasma et la salinité de l'eau d'abreuvement chez les caprins Arbia. Eltayeb (2000), au contraire, n'a pas trouvé d'effet significatif de l'augmentation de la salinité de l'eau sur les concentrations en protéines totales et en albumine sanguine chez des chèvres Nubiennes en saison estivale ; en revanche, en hiver, la salinité de l'eau a augmenté les concentrations en proteines totales et en albumine. Cet auteur attribue ces augmentations au rôle du sodium dans l'absorption des acides aminés de l'intestin et l'utilisation subséquente de ces derniers dans la formation des protéines plasmatiques.

La privation d'eau de $25 \%$ a augmenté significativement les concentrations sériques en protéines totales et en créatinine chez les chèvres Arbia, comparativement à l'hydratation normale. Plusieurs auteurs ont noté une augmentation des deux paramètres lors d'une épreuve de déshydratation, notamment Eltayeb (2000) chez les chèvres Nubiennes, Alamer (2005) chez des chèvres d'Arabie Saoudite, Aganga et al. (1988) chez des caprins Maradi, et Casamassima et al. (2016) chez des brebis Lacaunes. Cependant, une augmentation modérée du taux de créatinine plasmatique a été observée après trois jours de restriction hydrique chez le mouton Barki (Abdelatif et Ahmed, 1994).

La créatinine plasmique peut être utilisée comme un indicateur du taux de filtration glomérulaire (GFR) chez les animaux domestiques (Finco, 1997). L'accumulation de la créatinine dans le plasma pourrait être une conséquence de la réduction générale de l'excrétion urinaire durant la phase de restriction hydrique. Comme un ajustement à l'équilibre de l'eau pendant la phase de restriction hydrique, les animaux réduisent la filtration glomérulaire, ce qui entraîne une réduction du volume d'urine. L'augmentation de la créatinine plasmatique pourrait donc être liée au maintien de la fonction rénale à un niveau inférieur qui a pour conséquence une mauvaise élimination de la créatinine. La présente étude a révélé que la concentration en créatinine augmentait aussi avec le niveau de salinité de l'eau, ce qui corrobore les travaux d'Hadjigeorgiou et al. (2000) qui ont enregistré une augmentation de ce paramètre à mesure que la salinité d'eau d'abreuvement augmentait $(0,0,5,5,10$ et $20 \%)$.

Dans notre étude, le taux d'albumine sérique des caprins a été influencé par la salinité de l'eau, ainsi que par la privation partielle en eau des animaux. Il en est de même pour les chèvres Nubiennes pour lesquelles la déshydratation augmente significativement le taux d'albumine (Eltayeb, 2000).

Concernant l'urée sanguine, cette dernière a été affectée par la restriction hydrique enregistrant ainsi des valeurs situées à la limite 
supérieure de celles rapportées par Kaneko et al. (2008), cités par Kouamo et al. (2014). En revanche, ces valeurs, bien que décroissantes à mesure que l'eau devient saline, restent dans les normes de ces auteurs.

Une diminution significative du taux d'urée sérique chez les animaux recevant des valeurs croissantes de $\mathrm{NaCl}$ dans leur eau d'abreuvement a été observée comparativement aux animaux témoins. Ces résultats sont similaires à ceux de Eltayeb (2000) qui rapporte des concentrations sériques d'urée significativement faibles chez les chèvres Nubiennes ayant reçu une eau d'abreuvement contenant de grandes concentrations de $\mathrm{NaCl}(1,6 \%$ et $2 \%)$ par rapport aux chèvres ayant reçu une eau d'abreuvement avec des concentrations plus faibles de $\mathrm{NaCl}(0,8 \%$ et $1,2 \%)$. Ceci pourrait être attribué à une augmentation du GFR. Godwin et Williams (1986), et Meintjes et Engelbrecht (2004) signalent que le GFR est significativement plus élevé chez les chèvres et les moutons s'abreuvant d'eau à fortes concentrations de $\mathrm{NaCl}$ que chez ceux s'abreuvant avec de l'eau du robinet et à faibles concentrations de $\mathrm{NaCl}$. Les concentrations élevées de $\mathrm{NaCl}$ dans l'eau d'abreuvement a pour effet de rendre beaucoup plus disponible l'urée des tubules du néphron. Ceci pourrait, en outre, être attribué à une augmentation du transfert de l'urée au rumen. Meintjes et Engelbrecht (2004) rapportent que le passage d'urée au rumen est activé par l'apport d'eau d'abreuvement saline, et que cela peut avoir un effet sur les concentrations plasmatiques d'urée, ou éventuellement dans des conditions d'excès d'apport en sel, le rein ajuste le rapport de l'urée au sodium dans l'interstitium médullaire. Les résultats de notre étude chez les chèvres sont en accord avec les conclusions de Weeth et al. (1960) chez les génisses, et Meintjes et Engelbrecht (2004) chez les moutons.

Dans la présente étude, l'urémie a été significativement influencée par la restriction hydrique. Ainsi, les animaux du lot 4, partiellement privés d'eau d'abreuvement, ont présenté des valeurs d'urée sérique significativement supérieures à celles des autres lots. Cette augmentation serait due à l'hémoconcentration. Comme les protéines, l'urée jouerait un rôle important lors d'une restriction hydrique. Cette augmentation en urée pourrait être imputable partiellement à l'hormone antidiurétique (ADH). Olsson et Dahlborn (1989), cités par Eltayeb (2000), rapportent que la privation d'eau s'accompagne d'une augmentation de l'ADH chez les chèvres, ce qui minimiserait une perte d'urée puisque l'ADH favorise la réabsorption d'urée. En outre, cette situation pourrait être associée à une augmentation du catabolisme des protéines corporelles (Houpt, 2004, cité par Eltayeb, 2000).

Safari (2009) rapporte que les hyperurémies s'observent lors de néphrites aiguës et chroniques, dans l'insuffisance rénale et l'intoxication urémique. Une augmentation du niveau d'urée sanguine lors d'une restriction d'eau a déjà été signalée chez des chèvres (Aganga et al., 1988) et des bovins (Eltayeb, 2000). Maloiy et al. (1971), cités par Eltayeb (2000), signalent que la concentration d'urée diminue lorsque les moutons sont nourris avec un régime à faible teneur en protéines et limités dans leur consommation d'eau.

\section{CONCLUSION}

L'administration de $\mathrm{NaCl}$ dans l'eau d'abreuvement des caprins de race locale à des taux de 2 et $5 \mathrm{~g} / \mathrm{L}$ a augmenté les taux de créatinine, d'albumine, de cholestérol total et de protéines totales. Il en était de même après une épreuve de restriction hydrique qui, en outre, a augmenté les taux d'urée sanguine. L'augmentation de l'urée et de la créatinine chez les caprins suggèrerait une altération de la fonction rénale. Il serait intéressant d'étudier la réponse à long terme de l'effet du stress hydrique dû à une salinité excessive ou à une restriction d'eau sur les performances de production, le système endocrinien, et la production de lait et sa composition.

\section{REFERENCES}

AAVL (Association pour l'aménagement de la Vallée du Lot), 2006. Les systèmes d'abreuvement au pâturage ; Concilier production agricole et préservation des milieux aquatiques. Guide technique. Rivières Rance et Célé, Figeac, France, 3 p.

Abdelatif A.M., Ahmed M.M.M., 1994. Water restriction, thermoregulation, blood constituents and endocrine responses in Sudanese desert sheep. J. Arid Environ., 26: 171-180, doi : 10.1006/jare.1994.1021

Aganga A.A., Alabi O., Momoh M., 1988. Effect of water deprivation on nutrient digestibility, nitrogen retention, and water excretion in Yankasa sheep and Maradi goats. Niger. J. Anim. Prod., 15: 139-143

Alamer M., 2005. Physiological responses of Saudi Arabia indigenous goats to water deprivation. Small Rumin. Res., 63: 100-109, doi: 10.1016/j.smallrumres. 2005.02.004

Beede D.K., 2005. Evaluation of water quality and nutrition for dairy cattle. In: Proc. 7th Western Dairy Management Conf., 9-11 Mar. 2005, Reno, NV USA, 1-24

Belghiti M.L., Chahlaoui A., Bengoumi D., El Moustaine R., 2013. Etude de la qualité physico-chimique et bactériologique des eaux souterraines de la nappe plio-quaternaire dans la région de Meknès (Maroc). Larhyss J., 14 : $21-36$

Berthelot M.P.E., 1860. Chimie organique fondée sur la synthèse. Gallica, BNF, Paris, France p. 284

Casamassima D., Pizzo R., Palazzo M., D’Alessandro A.G., Martemucci G. 2008. Effect of water restriction on productive performance and blood parameters in Comisana sheep reared under intensive condition. Small Rumin. Res., 78: 169-175, doi: 10.1016/j.smallrumres.2008.03.014

Casamassima D., Vizzarri F., Nardoia M., Palazzo M., 2016. The effect of waterrestriction on various physiological variables in intensively reared Lacaune ewes. Vet. Med., 61 (11): 623-634, doi: 10.17221/144/2015-VETMED

El Moustaine R., Chahlaoui A., Bengoumi D., Rour E., Belghiti E., 2013. Contribution à la connaissance de la qualité bactériologique et physicochimique de l'eau des puits en élevage avicole dans la région de Meknes (Maroc). Science Lib, $5: 1-21$

Eltayeb E.E., 2006. Effect of salinity of drinking water and dehydration on thermoregulation, blood and urine composition in Nubian goats. Thesis, Master Vet. Sci. (Physiology), University of Khartoum, Sudan, 227 p.

Finco D.R., 1997. Kidney function. In: Clinical biochemistry of domestic animals, 5th Edn (Eds. Kaneko J.J., Harvey J.W., Bruss M.L.). Haracourt Brace, Delhi, India, 441-484

Godwin I.R., Williams V.J., 1986. Effects of intraruminal sodium chloride infusion on rumen and renal nitrogen and electrolyte dynamics in sheep. $\mathrm{Br}$. J. Nutr., 56 (2): 379-94, doi: 10.1079/BJN19860119

Gomaa S.A.M., 1996. Thermal stress and its relation to rumen function and some colorigenic hormones in sheep. PhD Thesis, Faculty of Agriculture, AlAzhar University, Cairo, Egypt

Gouaidia L., 2008. Influence de la lithologie et des conditions climatiques sur la variation des paramètres physico-chimiques des eaux d'une nappe en zone semi-aride, cas de la nappe de Meskiana, nord-est Algérien. Thèse Doct., Université Badji Mokhtar, Annaba, Algérie, 131 p.

Grech-Angelini S., 2007. Effets de la déshydratation sur le métabolisme énergétique et sur l'état corporel du dromadaire, Camelus dromedarius. Thèse, Ecole nationale vétérinaire, Toulouse, France, 121 p.

Hadjigeorgiou I., Dardamani K., Goulas C., Zervas G., 2000. The effect of water availability on feed intake and digestion in sheep. Small Rumin. Res., 37: 147-150, doi: 10.1016/S0921-4488(99)00142-X.

HCEFLCD, 2006. Etude diagnostique de la zone humide AL Massira-Faija, cercle d'EL Brouj et Cercle de Settat. HCEFLCD, Rabat, Maroc, 242

Jackson P.G.G., Cockcroft D.P., 2002. Laboratory reference values: Biochemistry; Appendix 3. Clinical examination of farm animals. Blackwell Science

King E.J., Wooton T.D.P., 1965. Determination of total protein in plasma or serum. In: Medical Biocemchemistry. Churchill, London, UK, 138-450

Kouamo J., Safari T., Miguiri K., Sandeu M.M., Sawadogo G.J., 2014. Etude de paramètres nutritionnels chez la chèvre sahélienne inséminée à l'œstrus induit (éponge de FGA et eCG). Livest. Res. Rural Dev., 26, www.lrrd.org/ |rrd26/3/koua26038.html 
Meintjes R.A., Engelbrecht H., 2004. Changes in the renal handling of urea in sheep on a low protein diet exposed to saline drinking water. Onderstepoort J. Vet. Res., 71 (3): 165-70, doi: 10.4102/ojvr.v71i3.255

Murray R.L., 1984. Creatinine. In: Clinical chemistry; Theory, analysis and correlation (Eds. Kaplan L.A., Pesce A.J.). Mosby, St. Louis, USA, 1247-1253

Olkowski A.A., 2009. La qualité de l'eau d'abreuvement du bétail : Guide de terrain relatif aux bovins, aux chevaux, à la volaille et aux porcs, $1^{\text {re }}$ édn. Ministère de l'Agriculture et Agroalimentaire, Canada, 185 p.

Richmond W., 1973. Preparation and properties of a cholesterol oxidase from Nocardia sp. and its application to the enzymatic assay of total cholesterol in serum. Clin. Chem, 19/12: 1350-1356

Rodier J., Legube B., Merlet N., 2009. L'analyse de l'eau, ge édn. Dunod, Paris, France, $1579 \mathrm{p}$.

Safari T., 2009. Evaluation de l'impact des paramètres protéiques et enzymatiques sur le taux de réussite de l'insémination artificielle caprine dans la région de Fatick au Sénégal. Thèse Doct., Ecole inter-Etats des sciences et médecine vétérinaires, Dakar, Sénégal, 90 p.

Sahi S., Afri-Bouzebda F., Bouzebda Z., Djaout A., 2018. Etude des mensurations corporelles de caprins dans le Nord-Est algérien. Livest. Res. Rural Dev., 30, www.Irrd.org/lrrd30/8/sameh30140.html
Silanikove N., 2000. The physiological basis of adaptation in goats to harsh environment. Small Rumin. Res., 35: 181-193, doi: 10.1016/S0921-4488 (99)00096-6

Trinder P., 1969. Determination of glucose in blood using glucose oxidase with an alternative oxygen acceptor. Ann. Clin. Biochem., 6: 24-25, doi: $10.1177 / 000456326900600108$

Weeth H.J., Haverland L.H., Cassard D.W., 1960. Consumption of sodium chloride water by heifers. J. Anim. Sci., 19 (3): 845-851, doi: 10.2527/ jas1960.193845x

Wilson A.D., 1975. Influence of water salinity on sheep performance while grazing on natural grassland and saltbush pastures. Aust. J. Exp. Agric. Anim. Husb., 15 (77): 760-765, doi: 10.1071/EA9750760

Young D.S., Friedman R.B., 2001. Effects of disease on clinical Laboratory tests, $5^{\text {th }}$ Edn. AACC, Washington DC, USA

Yousfi I., Ben Salem H., 2017. Effect of increasing levels of sodium chloride in drinking water on intake, digestion and blood metabolites in Barbarine sheep. Ann. INRAT, 90: 202-214, doi: 10.12816/0028688

Zabre Z.M., 2013. Détermination des paramètres biochimiques usuels chez les petits ruminants du Burkina Faso et leurs variations chez les sujets infectés naturellement par la trypanosomose. Thèse Doct., Université Cheikh Anta Diop, Dakar, Sénégal, 106 p.

\section{Summary}

Mehalaine Z., Chaker-Houd K., Ghamri A.N., Mebirouk-Boudechiche L., Matallah S. Effect of salinity and water restriction on blood metabolites of Arbia goats in Algeria

The study aimed to determine the effect of water stress on the blood metabolites of Arbia goats in Algeria. Four groups of ten billy goats each were randomly assigned to different watering regimes: group 1 (control) had free access to spring water (three liters), groups 2 and 3 received the same water enriched with two and five grams of sodium chloride per liter, respectively, and group 4 only had access to $25 \%$ of the amount of water given to the control group. At the end of the experimental period, blood samples were taken to measure total protein, creatinine, albumin, urea, total cholesterol and glucose. The various treatments increased plasma concentrations of albumin, creatinine, total protein and total cholesterol, as well as urea in water-restricted animals. However, they did not affect blood sugar levels. These results showed that short-term (12day) exposure of Arbia billy goats to salinization of drinking water or to water restriction affected the majority of their blood metabolites with the exception of glucose.

Keywords: goats, saline water, water deprivation, metabolites, Algeria

\section{Resumen}

Mehalaine Z., Chaker-Houd K., Ghamri A.N., Mebirouk-Boudechiche L., Matallah S. Efecto de la salinidad y de la restricción de agua en los metabolitos sanguíneos en cabras Arbia en Argelia

El objetivo del estudio fue determinar el efecto del estrés de agua en los metabolitos sanguíneos de cabras Arbia en Argelia. Cuatro grupos de diez machos cabríos fueron asignados al azar a diferentes regímenes de agua: grupo 1 (control) tuvo libre acceso a una fuente de agua (tres litros), grupos 2 y 3 recibieron la misma agua, enriquecida con dos y cinco gramos de cloruro de sodio por litro respectivamente y el grupo 4 sólo tuvo acceso a $25 \%$ de la cantidad de agua ofrecida al grupo control. Al final del periodo experimental, se tomaron muestras de sangre para medir la proteína total, creatinina, albúmina, urea, colesterol total y glucosa. Los diversos tratamientos aumentaron las concentraciones en plasma de albúmina, creatinina, proteína total y colesterol total, así como urea en los animales con restricción de agua. Sin embargo, no afectaron los niveles de azúcar sanguíneo. Estos resultados muestran que una exposición corta (12 días) en los machos cabríos Arbia a una salinización del agua de beber o restricción de agua afectó la mayoría de los metabolitos sanguíneos con excepción de la glucosa.

Palabras clave: caprino, agua salina, privación del agua, metabolitos, Argelia 